\title{
Representaciones científicas en la enseñanza de la química: un reconocimiento de los niveles representacionales de la materia
}

\author{
Scientific representations of chemistry teaching: a \\ recognition of the representational levels of matter
}

\author{
Yolima Ivonne Gómez Mora1 \\ Milba Dayaliz Ballesteros Vásquez ${ }^{2}$ \\ Quira Alejandra Sanabria Rojas ${ }^{3}$
}

\section{Resumen}

El presente trabajo se centra en la caracterización de niveles de representación científica de los estudiantes de séptimo, octavo y décimo del Instituto Pedagógico Nacional. Dichos niveles se rastrearon por medio de la implementación de una secuencia de actividades propuestas en la clase de Biología y Química respectivamente, asociados a conceptos sobre las sustancias, sus propiedades y las reacciones químicas. Los resultados se examinan a través del empleo de tres categorías de análisis referidos a los niveles de representación macroscópico, microscópico y simbólico. Esta evaluación se hizo en función de la comprensión y uso discursivo de términos sobre la discontinuidad de la materia, representaciones gráficas, matemáticas y verbales, entre otros. Se destaca que los grupos analizados hacen un uso del discurso con una función principal: comunicar sus ideas, aunque en la medida que deje de ser un análisis consciente, difícilmente el estudiante comprende por qué en química el cambio de las sustancias requiere de más de un tipo de representación. Por lo tanto, en consonancia con el objetivo general del proyecto, es válido afirmar que los estudiantes en las clases de ciencias usan y representan el comportamiento de la materia en todos los niveles

1 Profesor en formación Universidad pedagógica Nacional. Correo electrónico: dqu_ygomez079@pedagogica. edu.co

2 Profesor en formación Universidad pedagógica Nacional. Correo electrónico: dqu_mdballesterosv19o@pedagogica.edu.co

3 Asesora de práctica Programa Licenciatura en Química Universidad Pedagógica Nacional 
de representación; independientemente que los reconozcan, la responsabilidad del profesor para que mejore la comprensión de los fenómenos químicos está articulada en gran medida con la capacidad que tenga para mediar en los discursos a propósito de las condiciones que suscitan los cambios de las sustancias.

\section{Palabras clave}

enseñanza de la química; niveles de representación en química; lenguajes químicos; modelos mentales; modelos conceptuales.

\section{Abstract}

The present work focuses on the characterization of levels of scientific representation of the seventh, eighth and tenth students of the National Pedagogical Institute. These were tracked through the implementation of a sequence of activities proposed in the biology and chemistry class respectively. Associated with concepts about substances, their properties and chemical reactions. The results are examined through the use of three categories of analysis referring to the levels of macroscopic, microscopic and symbolic representation. This evaluation was made based on the understanding and discursive use of terms on the discontinuity of the subject, graphic, mathematical and verbal representations, among others. It is emphasized that the analyzed groups make use of the discourse with a main function; communicate their ideas, although to the extent that it ceases to be a conscious analysis, the student hardly understands why in chemistry the change of substances requires more than one type of representation, therefore, in line with the overall objective of the project is valid affirm that students in science class use and represent the behavior of the subject at all levels of representation, regardless of whether they recognize them, the responsibility of the teacher to improve the understanding of chemical phenomena is largely related to the ability that has to mediate the speeches with regard to the conditions that cause the changes of the substances.

\section{Keywords}

Teaching chemistry, levels of representation in chemistry, chemical languages, mental models, conceptual models.

\section{Introducción}

Las ciencias son el resultado de pensar e imaginar el mundo; una representación en general es una expresión de la cognición humana que se expresa como notación, signo o conjunto de símbolos que refiere a las características que describen un objeto, condición, fenómeno natural, el cual capta el interés del individuo o de un grupo de ellos, este se encuentra en el mundo exterior del individuo o en su mundo interior, lo que se denomina imaginación. Por ejemplo, la palabra muñeca o el dibujo de una muñeca son representaciones externas que nos permiten evocar el objeto muñeca en su ausencia, de igual forma Johnson-Laird (1983); Moreira, Greca, y Rodríguez Palmero (2002), mencionan que las representaciones mentales son maneras de "representar" internamente lo que el individuo percibe por medio de los sentidos. Lo anterior sugiere una necesidad urgente para reflexionar cómo los estudiantes de educación básica y secundaria construyen representaciones científicas a propósito de las afirmaciones de Johnson-Laird (1983), quien propone que el mundo de las ideas científicas se construyen sobre tres tipos de representaciones mentales a nivel estructural: la primera, representaciones proposicionales o cadenas de símbolos, las cuales no están constituidas por palabras; sin embargo, pueden traducirse en términos; por otro lado, captan el contenido abstracto, que sería codificado es decir, el lenguaje de la mente, siendo capaz de admitir más de una representación para el mismo objeto de estudio. Dichas representaciones proposicionales pueden ser falsas o verdaderas dependiendo de las características que como criterios de validez usen en la interpretación.

El segundo tipo lo constituyen los modelos mentales, que representan un estado importante de las cosas correspondiente a un mundo observable a simple vista o imaginario. A diferencia de la primera 
categoría, los modelos mentales no poseen una estructura sintáctica, por lo que son manipulables de manera controlada por las mismas dimensiones del modelo; por ejemplo, inflar o desinflar una bomba, el sujeto puede explorar este modelo en su mente. Finalmente, las imágenes, para Johnson-Laird (1983), son producto tanto de la percepción como de la imaginación, correspondiente a características de los objetos, todas ellas necesarias para poder explicar las maneras en las que las personas, representan, razonan, hacen inferencias, comprenden lo que los otros hablan y entienden el mundo real. Las imágenes son específicas y también sujetas a transformaciones como expansiones, rotaciones y traslaciones. Sin embargo, estos son incompletos, no son bien definidos y por tanto son confusos, son inestables, ya que las personas tienden a olvidar detalles; no son científicos, pues refleja en ocasiones las creencias de los sujetos ante un sistema físico, de modo que reflejan poca estabilidad y baja capacidad predictiva (Moreira, Greca y Rodríguez Palmero, 2002).

A diferencia de los modelos mentales, Norman (1983) menciona que los modelos conceptuales son aquellos inventados y diseñados por investigadores, ingenieros, arquitectos y profesores, utilizados para facilitar la comprensión de sistemas físicos que se han hecho como objeto de estudio desde el interés particular de una comunidad, en este caso de especialistas. La siguiente cita agrupa la explicación sobre el modelo conceptual que se adoptó en este trabajo.

Son representaciones externas, compartidas por una determinada comunidad y consistentes con el conocimiento científico que esa comunidad posee. Estas representaciones externas pueden materializarse en forma de formulaciones matemáticas, verbales o pictóricas, de analogías o de artefactos materiales [...], La analogía entre el átomo de Rutherford y el sistema solar [...], son ejemplos de modelos conceptuales. Independientemente de la forma que puedan adquirir los modelos conceptuales de disciplinas como la Física (por ejemplo, un conjunto de fórmulas matemáticas y de leyes empíricas), la Química (por ejemplo, una serie de ecuaciones químicas y de estructuras geométricas) [...], todos ellos tienen en común que son representaciones simplificadas e idealizadas de objetos, fenómenos o situaciones reales, pero son precisos, completos y consistentes con el conocimiento científicamente aceptado. (Moreira, Greca y Rodríguez Palmero, 2002, p. 10).

Como los profesores de ciencias tenemos responsabilidad social con respecto al tipo de ciencias que se enseña, se propuso desde el marco de referencia ya mencionado el siguiente objetivo durante el desarrollo de la práctica pedagógica y didáctica: evaluar y caracterizar los niveles de representación científica de un grupo de estudiantes del Instituto Pedagógico Nacional (IPN de aquí en adelante), en relación con la representación macroscópica, microscópica y simbólica (Ordenes, Arellano, Merino y Jara, 2014), que se desarrollan cuando se abordan conceptos como átomo, elemento, molécula, compuesto, sustancia, cambio físico, por medio de la construcción y aplicación de una secuencia de actividades diseñadas para la asignatura. Esta indagación se hizo con el propósito de responder a la pregunta de investigación: ¿cómo los estudiantes conocen y relacionan los niveles de representación macroscópica, microscópica y simbólica en la enseñanza de la química cuando se abordan conceptos como elemento, sustancia, mezcla, reacción química, entre otros?

Quizás uno de los elementos detonadores para buscar la categorización de las representaciones científicas tiene que ver con afirmaciones como la siguiente: los estudiantes de secundaria no asocian las fórmulas químicas con el modelo de discontinuidad de la materia (Galagovsky, Rodriguez, Nora y Morales, 2003), que a nuestro modo de ver es una situación recurrente a la hora de encontrar explicaciones sobre las razones por las cuales los estudiantes difícilmente logran avances en las comprensiones teóricas de cómo se producen las reacciones, que como fenómeno, explican la trasformación de las sustancias (Maloney y Friedel, 1991). Como se ha encontrado en la literatura consultada, los estudiantes de secundaria por lo general logran construir representaciones de nivel macroscópico con facilidad, el cual se encuentra asociado a las características que es 
posible identificar por observación directa durante los experimentos propuestos en clase. Llamó la atención que era más difícil para los estudiantes de grado décimo elaborar representaciones de las reacciones de nivel microscópico con respecto a las representaciones macroscópicas. A diferencia de lo estudiantes de grado séptimo quienes pudieron representar los cambios químicos acudiendo a modelos macroscópicos combinados con representaciones microscópicas toda vez que para ellos fue más fácil imaginar la representación microscópica de las sustancias desde la comprensión del fenómeno de discontinuidad de la materia. Los autores ya mencionados afirman que la representación científica de la discontinuidad de la materia que logran establecer los estudiantes está estrechamente relacionada con las experiencias de laboratorio, pero sobre todo con la oportunidad que tienen los grupos de trabajo para reflexionar sobre lo observado, el uso de los dibujos que como analogía logran la construcción de presunciones sobre las condiciones que determinan el comportamiento de las sustancias, las cuales, a manera de partículas, interactúan entre sí, y esta actividad depende de unas condiciones que la delimitan. Para revisar la cercanía de lo documentado en un curso real, se propuso una serie de actividades que se ejecutaron en función de la metodología que se describe a continuación.

\section{Metodología}

La investigación se realizó en el Instituto Pedagógico Nacional ubicado en la localidad de Usaquén, con estudiantes de grado séptimo (702-704), octavo (803-804) y decimo (1003-1004), cuyas edades oscilan entre los 11 y 16 años y quienes cursan la asignatura Química. Los grados 1003 y 1004 se caracterizan por tener énfasis en artes y biología respectivamente. Para la construcción y aplicación de la propuesta de intervención, la metodología del trabajo constó de las siguientes etapas:

- Revisión de la literatura, elaboración del marco de referencia y planteamiento de objetivos.

- Diseño y construcción de actividades teniendo en cuenta los contenidos a desarrollar en cada uno de los grados (séptimo, octavo y décimo) en la asignatura Química.

- Implementación de actividades en el aula de clase, recolección y tratamiento de resultados obtenidos.

- Construcción de categorías de análisis por medio de una matriz para la evaluación de los resultados obtenidos en el desarrollo de las actividades propuestas, análisis de resultados y conclusiones.

A continuación, se describen cada una de las actividades implementadas durante la realización del proyecto.

Tabla 1. Descripción de actividades desarrollas por los estudiantes los grados séptimo, octavo y décimo del Instituto Pedagógico Nacional

Séptimo Titulo Actividad : Reconocimiento de las representaciones científicas en la enseñanza de la química.

En la actividad se da a conocer al estudiante los tres niveles de representación en química según Johnstone (1982) —el nivel macroscópico, el nivel microscópico y el nivel simbólico—, al hacer uso de analogías que permitan entender al estudiante cada uno de los niveles de representación. Enseguida el estudiante realiza dibujos de las representaciones científicas en la química desde objetos que se encuentran en su entorno. Para la anterior actividad se realiza un trabajo complementario que consiste en la observación de diferentes sustancias accesibles dentro del hogar, como son: el agua, el azúcar y la sal. A partir de la observación se realiza la descripción de las propiedades organolépticas de cada una y por medio del dibujo se hace referencia al estado inicial de cada sustancia. Además, el estudiante debe proponer un modelo de cómo cree que es la conformación de las moléculas, a partir de la anterior representación microscópica se procede a proponer una fórmula que describa el nivel simbólico, el cual debe ser corroborada realizando una consulta bibliográfica. 


\begin{tabular}{|c|c|}
\hline Octavo & $\begin{array}{c}\text { Título Actividad : La química cotidiana de los elementos. } \\
\text { Se pretende que el estudiante por medio de dibujos exprese dónde puede encontrar en su cotidianidad } \\
\text { los diferentes elementos químicos enunciados en la actividad y de acuerdo con las respuestas establecer el } \\
\text { nivel de representación de la materia que emplean sin intervención del docente. }\end{array}$ \\
\hline Decimo & $\begin{array}{r}\text { Título Actividad Introducción a las reacciones químicas, conceptos sobre materia. } \\
\text { Para la actividad se espera que los estudiantes, a través de representaciones macroscópicas y microscópi- } \\
\text { cas, reconozcan conceptos básicos de la materia como átomo, molécula, compuesto, sustancia y mezcla. } \\
\text { Así mismo identifiquen cambios físicos y químicos de la materia por medio de ejemplos cotidianos. Por } \\
\text { último, introducir al estudiante en representaciones simbólicas de ecuaciones químicas a través de la inter- } \\
\text { pretación de representaciones a nivel microscópico de la materia. }\end{array}$ \\
\cline { 2 - 3 } & $\begin{array}{c}\text { Título Actividad.: Clases de reacciones químicas. Para la actividad se propone que el estudiante complete } \\
\text { las representaciones faltantes correspondientes a los niveles macroscópico, microscópico y simbólico de } \\
\text { cada uno de los ejemplos planteados según el tipo de reacción química. }\end{array}$ \\
\hline
\end{tabular}

Fuente: elaboración propia.

La información recolectada por medio de las actividades descritas anteriormente se analizó acorde a las categorías de la matriz referentes a los niveles de representación científica utilizados en química (tabla 2), con el fin de evaluar la relación representacional macroscópica, microscópica y simbólica de los estudiantes de los grados séptimo, octavo y decimo del Instituto Pedagógico Nacional, al abordar conceptos básicos de la materia como son átomo, molécula, entre otros, fundamentales en el aprendizaje de la química.

Tabla 2. Matriz de análisis para las categorías macroscópico, microscópico y simbólico

\begin{tabular}{|c|c|c|}
\hline $\begin{array}{l}\text { Nivel de representación de las ciencias } \\
\text { o categoría }\end{array}$ & Subcategoría & Descriptor \\
\hline \multirow{3}{*}{$\begin{array}{l}\text { Microscópico } \\
\text { (abstracto) }\end{array}$} & Partícula. & Discontinuidad de la materia. \\
\hline & Cambio de estado. & Cambio físico y cambio químico. \\
\hline & Representación simbólica. & Lenguaje verbal. \\
\hline \multirow{3}{*}{$\begin{array}{c}\text { Macroscópico } \\
\text { (Depende de la experiencia con el } \\
\text { medio) }\end{array}$} & $\begin{array}{l}\text { Características organolépticas } \\
\text { (Olor, sabor, tamaño, dureza, textura, } \\
\text { entre otros). }\end{array}$ & Propiedades físicas. \\
\hline & Términos cotidianos. & $\begin{array}{l}\text { Lenguaje que expresa el comporta- } \\
\text { miento de la materia. }\end{array}$ \\
\hline & Representación simbólica. & $\begin{array}{l}\text { Cercano a lo que se percibe por medio } \\
\text { de los sentidos. }\end{array}$ \\
\hline \multirow{3}{*}{ Simbólico } & Lenguaje gráfico. & Dibujos, esquemas, analogías. \\
\hline & Lenguaje verbal. & Letras, números, términos. \\
\hline & Lenguaje matemático. & Ecuaciones, formulas. \\
\hline
\end{tabular}

Fuente: elaboración propia. 


\section{Resultados y discusión}

A continuación, se presentan los resultados que se destacan en el proyecto de investigación:

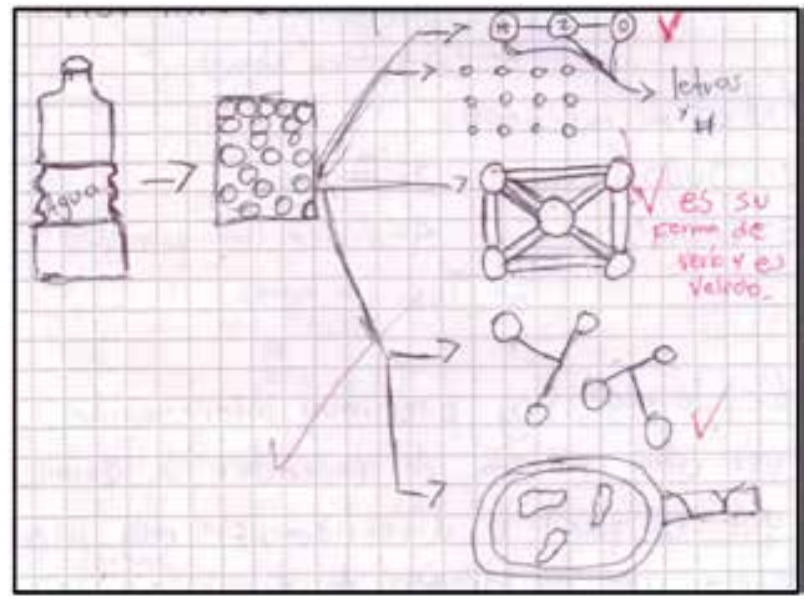

Figura 1. Representación de la materia discontinua, en este caso para la sustancia agua

Fuente: trabajo presentado por estudiante grado séptimo
Grado séptimo: sin proporcionar a los estudiantes un modelo científico de la molécula del agua, los niños proponen sus modelos representacionales a nivel microscópico; en la parte superior derecha de la figura 1 se observa una representación de la molécula del agua involucrando la presentación simbólica; también se detalla cómo cada individuo puede imaginarse a nivel simbólico la molécula, lo cual es un resultado favorable, porque de esta manera se está haciendo inclusión del dicho nivel representacional en el aula de clase. Por otro lado, se observa el icono de una lupa y en su interior el objeto particulado, esto visto teóricamente significa que no hay una representación discontinua de la materia si no una representación semi-particulada en la que Galagovsky menciona que en el triángulo de Johnstone debería tenerse en cuenta, ya que el estudiante como sujeto inexperto sufre una transición entre en nivel representacional macroscópico y el microscópico un nivel representacional intermedio, erróneo denominado semi-particulado (Galagovsky, Rodriguez, Nora y Morales, 2003).

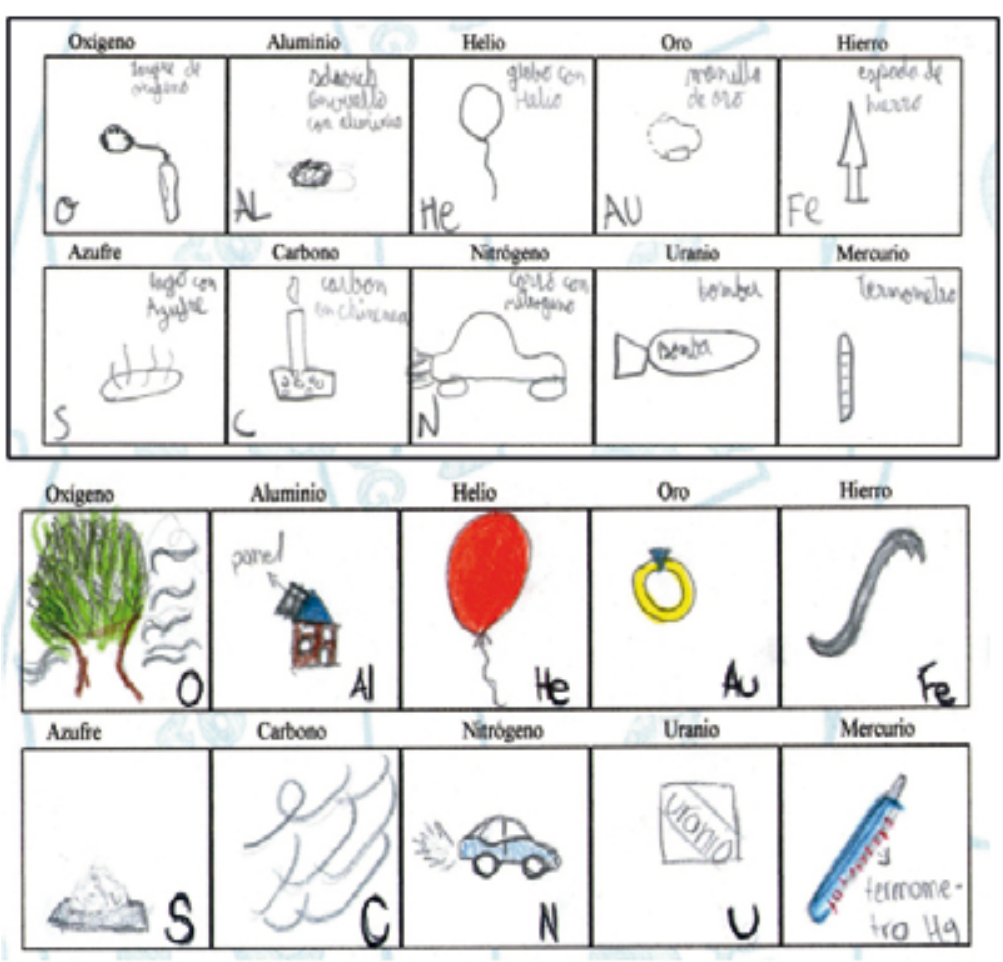

Figuras 2 y 3 . Algunos dibujos de los niños de octavo

Fuente: trabajo estudiante de grado octavo 
Grado octavo: los resultados obtenidos con los niños demuestran la necesidad de la inclusión del nivel representacional microscópico, ya que durante la intervención educativa no se hizo explicito este nivel y se esperaba que los estudiantes contestaran iconográficamente la siguiente pregunta: ¿En dónde se pueden encontrar los elementos Oxígeno, Aluminio, Helio, Oro, Hierro, Azufre, Carbono, Nitrógeno, Uranio y Mercurio en la vida cotidiana? Como se puede evidenciar en las figuras 2 y 3, los estudiantes no tienen la noción de elemento, pues si no, hubiesen suministrado la frase que enuncia dicha imagen, la interpretación de los resultados sería compleja. Como menciona Norman (1983), los modelos mentales son incompletos, no son bien definidos y por tanto son confusos, además de ser inestables, pues las personas suelen olvidar detalles. Un ejemplo de ello es el dibujo sin color que representa oxígeno o el dibujo de termómetro, pues sin la frase no se pensaría que fuera.

Grado décimo: se puede decir que la su mayoría de estudiantes presentan dificultad al relacionar los niveles de representación de forma coherente, lo cual se evidenció en la actividad 2, en uno de los apartados (imagen 4), ya que se muestra a nivel microscópico la ecuación química señalada simbólicamente, para que a partir de la información dada dibujaran la representación macroscópica; a pesar de la iconografía propuesta por el estudiante esta no corresponde correctamente.

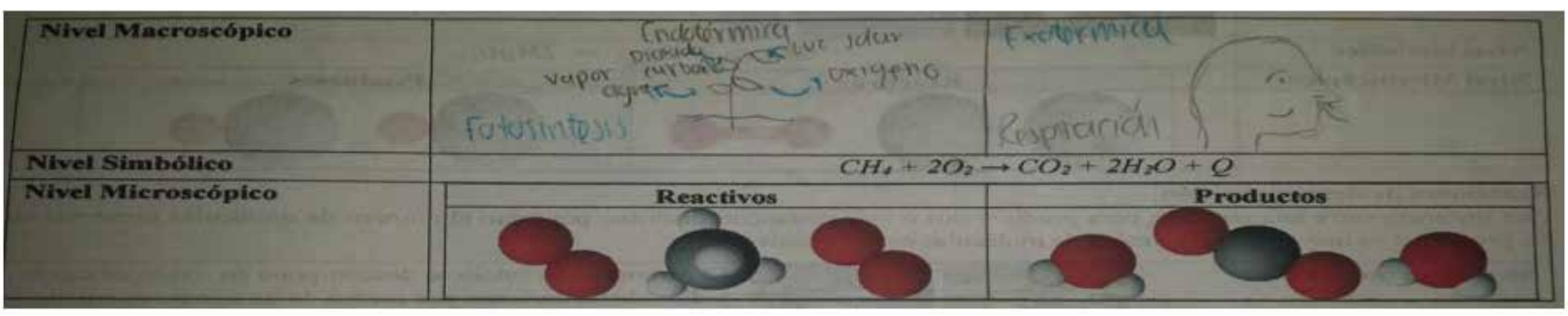

Figura 4. Reacciones endotérmicas y exotérmicas

Fuente: Trabajo presentado por estudiante de grado décimo

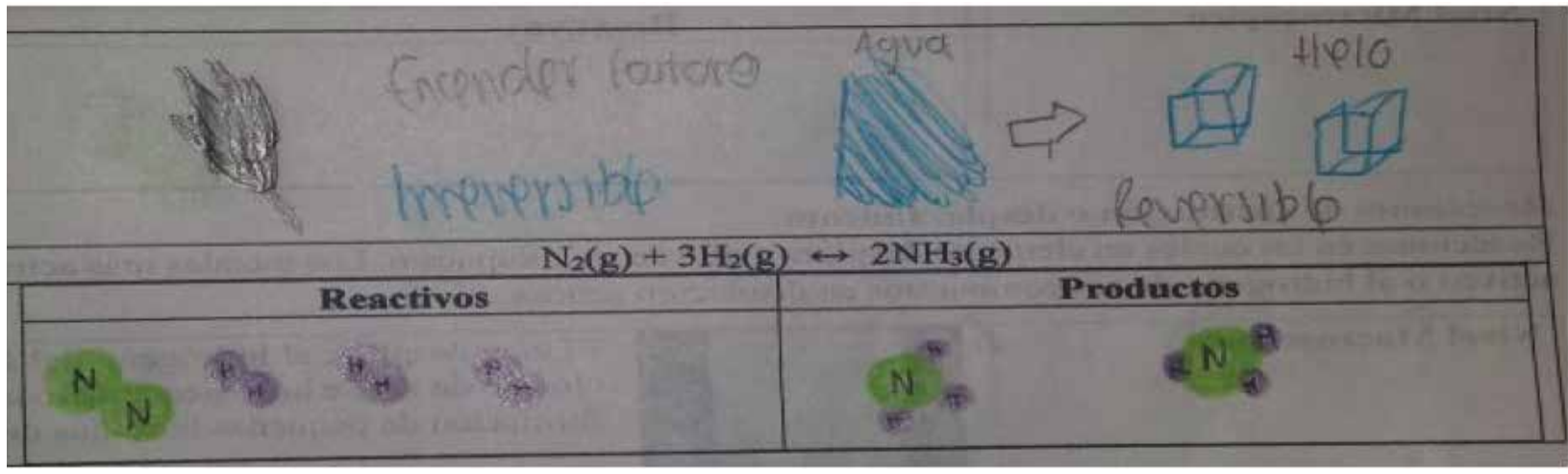

Figura 5. Reacciones reversibles e irreversibles

Fuente: trabajo presentado por estudiante de grado décimo

En la figura 5 se observa, por ejemplo, que los estudiantes se aproximaron significativamente a las representaciones científicas que se encuentran en los libros de texto sin que hubiera intervención anticipada por parte del profesor. En el ejemplo propuesto también es posible identificar que el acto de encender un fósforo lo clasifican como proceso irreversible, aunque no logra establecer 
un argumento, toda vez que lo escrito afirma que el cambio de estado del agua es reversible. En este caso, debido a lo representado por el estudiante frente al fenómeno analizado, se afirma que el estudiante aún no tiene claro el concepto de reacción ya que menciona un ejemplo relacionado a propiedades físicas, por otro lado, las representaciones propuestas no son coherentes con la representación simbólica y microscópica propia de una reacción. Luego de culminar el análisis de los documentos construidos por la muestra seleccionada se llegaron a las siguientes conclusiones generales:

- En términos representacionales para el grado décimo, los estudiantes hacen una aproximación al modelo discontinuo de la materia; sin embargo, en algunas representaciones no abandonan el modelo continuo de la materia, y de otro lado, hacen una correspondencia sin mucha dificultad entre la representación simbólica y la representación iconográfica, mientras que al tratar de relacionar aspectos teóricos similares, como los estados de agregación de la materia, en los grados octavo a quienes no se les desarrolló un trabajo en particular con el modelo de discontinuo de la materia, muestran un modelo continuo de esta, visto a través de los dibujos. Por lo tanto, se puede decir que ellos tienen un modelo continuo de la materia, ejemplificado a partir de situaciones que son conocidas en lo cotidiano.

- Comparado con los resultados de los estudiantes de séptimo, con los que se desarrolló una intervención educativa a través el modelo discontinuo, cuando se hicieron preguntas semejantes, se encontró que las representaciones propuestas por los ellos son cercanas al modelo discontinuo de la materia, lo que sugiere un mejor nivel de abstracción.

- Con la actividad 2, "Clases de reacciones químicas", se pudo evidenciar que los estudiantes difícilmente plantean una representación macroscópica de un fenómeno a partir de una representación simbólica, como se propuso en la reacción de desplazamiento, ya que difícilmente han establecido relaciones entre los marcos teóricos y los fenómenos propuestos.

- Por otra parte, es necesario hacer la inclusión consiente del modelo de discontinuidad de la materia en el aula de clase, para la representación científica de las sustancias y entender el comportamiento de las mismas en los niveles macro y microscópico. En la medida que deje de ser un análisis consciente, difícilmente el estudiante comprende por qué en química el cambio de las sustancias requiere de más de un tipo de representación, y por ello, en consonancia con el objetivo general del proyecto, es válido afirmar que los estudiantes en la clase de ciencias usan y representan el comportamiento de la materia en todos los niveles de representación, independientemente de su reconocimiento. La responsabilidad del profesor para que mejore la comprensión de los fenómenos químicos está articulada en gran medida con la capacidad que tenga para intervenir en los discursos a propósito de las condiciones que suscitan los cambios.

\section{Referencias}

Johnson-Laird, P. N. (1983). Mental Models: Towards a Cognitive Science of Language, Inference. and Consciousness. Cambridge, MA: Harvard University Press.Galagovsky, L., Rodríguez, M., Nora, S. y Morales, L. (2003). Representaciones mentales, lenguajes y códigos en la enseñanza de ciencias naturales, un ejemplo para el aprendizaje del concepto de reacción química a partir del concepto de mezcla. Enseñanza de las ciencias, 21(1), 107-121. Recuperado de www.raco.cat/index.php/ Ensenanza/article/download/21898/21731

Galagovsky, L. y Giudice, J. (2015). Estequiometria y ley de conservación de la masa: una relación a analizar desde la perspectiva de los lenguajes químicos. Ciênc. Educ., Bauru, 21(1), 85-99. doi: http://dx.doi. org/10.1590/1516-731320150010006 
Johnstone (1982). Macro and microchemistry. School Science Review, 64, 377-376.

Maloney, D. P. y Friedel, A. W. (1991). Students'difficulties with subscripts in chemical formulas. Comunicación presentada a National Association for Research in Science Teaching Conference. Fontana, WI.

Moreira, M., Greca, I., y Rodríguez Palmero, M. (2002). Modelos mentales y modelos conceptuales en la enseñanza y aprendizaje de las ciencias. Educação em Ciências, Porto Alegre, 2(3), 37-57. Recuperado de https://www.if.ufrgs.br/ moreira/modelosmentalesymodelosconceptuales.pdf

Norman, D.A. (1983). Some observations on mental models. In D. Gentner y A.L. Stevens (Eds.), Mental models. Hillsdale, NJ: Lawrence Erlbaum Associates.

Ordenes, R., Arellano, M., Merino, C. y Jara, R. (2014). Representaciones macroscópicas, submicroscópicas y simbólicas sobre la materia. Educación química, 1(25), 46-55. Recuperado de https:// www.researchgate.net/profile/Cristian_Merino_ Rubilar/publication/264436566_Representaciones_macroscopicas_submicroscopicas_y_simbolicas_sobre_la_materia/links/53df85160cf27a7b83068175.pdf 